\title{
Influence of efficient, multicomponent, polyfunctional, physiologically active (nano) chips with herbicide activity on rice crop growth, development, yield and on weed growth inhibition
}

\author{
N. Voropaeva ${ }^{1}$, V. Karpachev ${ }^{1}$, V. Varlamov ${ }^{2}$, O. Figovsky ${ }^{3, *}$ \\ ${ }^{1}$ All-Russian Rapeseed Research Institute (ARRI), Lipetsk, Russia \\ 2 «Bioengineering» center RAS, Moscow, Russia \\ ${ }^{3}$ Polymate Ltd.-INRC, Migdal Ha'Emeq, Israel \\ *E-mail address: figovsky@netvision.net.il
}

\begin{abstract}
Using physiologically active, complex, polyfunctional, multicomponent (nano) systems (nano) chips in the pre-sowing rice treatment (nano) technology allows precise seeding; enhancing field seed germination; reducing plant morbidity rate considerably or even deleting disease incidents; increasing plant adaptability to unfavorable environmental conditions; expanding crop yield; improving products quality by not using toxic chemical means of plant protection; intensifying competitive ability of agricultural products as well as assuring environmental safety in crop cultivation regions. Nutrition micro- and mesoelements, as also intense activity plant growth regulators and other physiologically active substances, depending on the crop variety, cultivation conditions and possible risks, can be added to such pre-sowing treatment (nano) technology.
\end{abstract}

Keywords: Nanotechnologies; Agricultural; Seeds; Rice; Biopesticides; Immunomodulators; Elicitors; Herbicide; Nanochips

\section{INTRODUCTION}

In foreign countries a modified vermiculite that we used as (nano) matrixes is called a yield mineral and the Japanese call it a therapeutic mineral [1].

Agrovermiculite prevents mould, fungi and pests development. All these and a lot of other characteristics make it an effective and absolutely environmentally clean addition as the carrier of various physiologically active substances. Agrovermiculite consists of separate particles (flaky structure) that have a layered form and contain millions of air bubbles. Its presence improves the structure of the soil, increasing the amount of air in 2-3 times, which is highly favorable for plants development. 


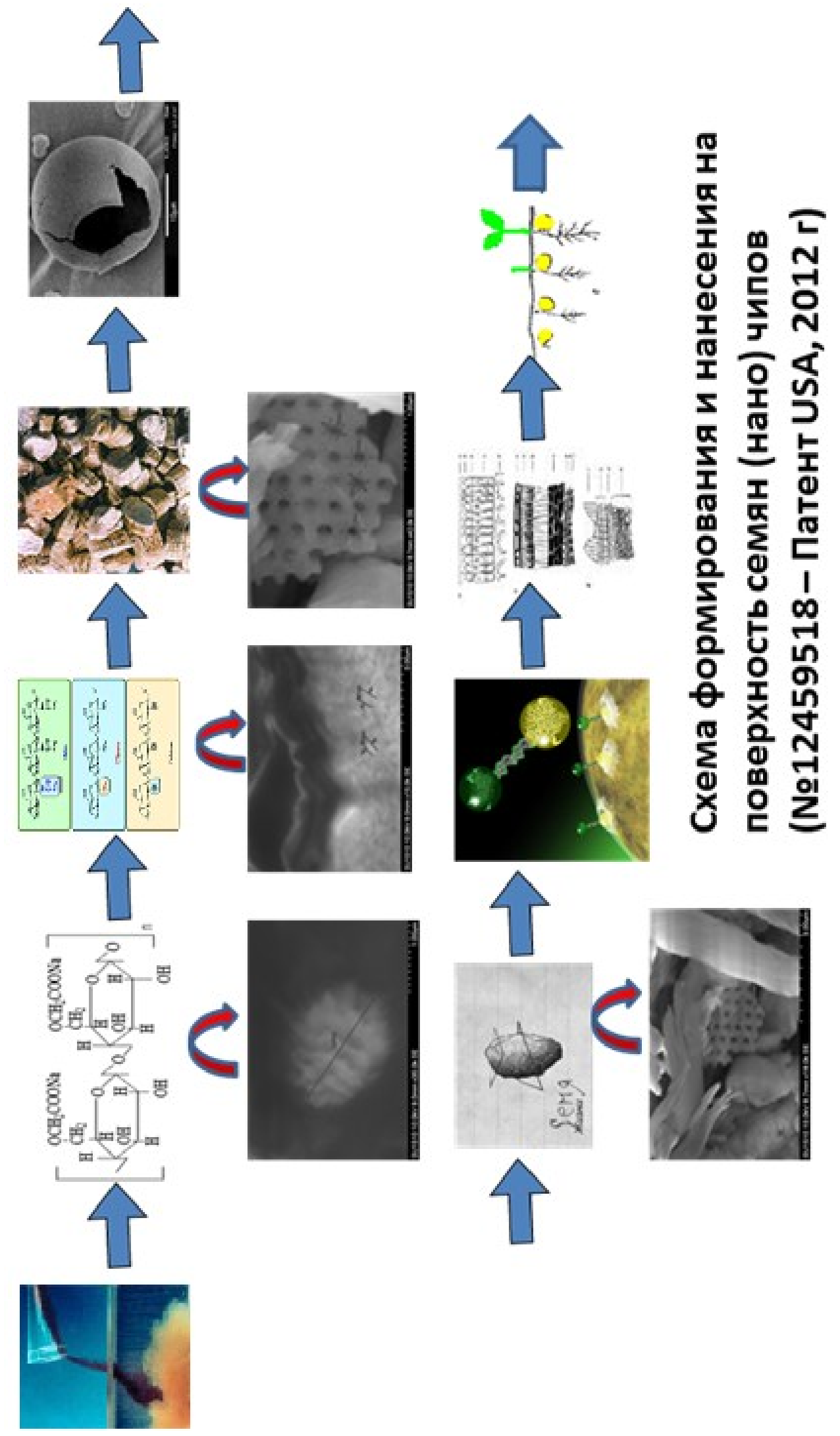

Fig. 1. Pre-sowing seed treatment model. 
Introducing this modified vermiculite to the contaminated soil as an active absorber (sorbent) of various chemical compounds, heavy metal ions and radionuclides, enables environmentally safe agricultural products manufacturing. Agrovermiculiteinstantly absorbs liquid fertilizers and then gives it gradually back to plants during several months or even years. Moreover, a high content of magnesium, potassium and other entries of the periodic table in the agrovermniculite (plant nutrition micro-, meso-, macroelemets) makes it a independent carrier of valuable microelements. A modified vermiculite can absorb moisture about $400-600 \%$ of his own weight, gradually giving it to the plant root system.

Decreasing nutrients wash-out from the soil that contains agrovermiculite considerably reduces fertilizer expenditures per cultivated area unit. Adding agrovermiculite into the soil provides the reduction of susceptibility to diseases. Currently, as is known, for plant protection against phytopathogens - pests chemical plant protection means are used that are often toxic and phytotoxic for the hematothermal. This may lead to unwelcome environmental changes and also to residue pesticide accumulation in the soil and agricultural products. As we know, internationally a particular importance is being attached to the development of "green" technologies, based on the usage of environmentally safe means and methods of plant protection (including biopesticides - preparations, obtained from natural sources). Thus, today the development of environmentally safe means of plant protection based on natural non-toxic compounds is a primary goal.

Throughout the process, special attention is devoted to the creation of environmentally safe technologies, in particular to (nano) technologies, for the agricultural sector, including pre-sowing seed treatment. One of the novel concepts of (nano) technology implementation is creating polyfunctional, multicomponent, biologically active (nano) chips for pre-sowing seed treatment (Fig.1). Such (nano) chips include carriers based on natural mineral derivatives with specified pore sizes and also include physiologically active substances (with biopesticides of natural origin and other low-toxic and non-toxic substances) in the form of water solutions, colloidal systems, fine-dispersed suspensions and emulsions for filling carriers' (nano) pores.

In our research we used natural polysaccharide derivatives (cellulose and chitosan) and modified minerals (modified vermiculite) as the carriers of physiologically active substances, we also used oligoaminosaccharid AgroHit, the elicitor that increases plant adaptability to adverse environmental aspects, as biopesticides with growth-regulating activity [2-5]. As a weed control means we used herbicide Rainbow.

\section{DISCUSSION}

In comparison with the control variant rice seed treatment with the developed (nano) systems led to a slight decrease of germination energy and seed germination ability due to herbicide inhibiting effect on rice shoots growth and development, for being a part of (nano) chip this herbicide has coated the seed surface. Both on the $10^{\text {th }}$ and on the $15^{\text {th }}$ sprouting day in the same variants, an increase of weed-control degree has been noted (barnyard-grass and sedge species).

The (nano) carriers of physiologically active substances, modified vermiculite and cellulose derivatives included the (nano) chip structure, facilitated in-vitro germination increase, rice shoots' stem and root length growth and also wet and dry weights increase.

The conducted research works allowed detecting most favorable (nano) structures of multicomponent, polyfunctional (nano) chips based on physiologically active substances, which had been used for pre-sowing seed treatment in the field experiments. 
The treated seeds have been sown during our field experiment. Germination capacity, weed plants number, plant stand density, stem and root length, wet and dry plant weight, efficiency of herbicide effect on weeds (barnyard-grass and sedge species) on 15, 30, 45 and $60^{\text {th }}$ day along with biometric data and rice crop yield indexes have been determined.

The small-plot field experiments have determined an increase of rice plants standing density on the shoots stage in several variants (where the seeds have been treated with developed multicomponent, physiologically active, polyfunctional (nano) chips with herbicide activity) as compared with the control variant (the seeds have not been treated). This can be probably due to regulation of water ingress speed and physiological and biochemical processes on the initial stages of its germination, which stems from the nature of matrixes carriers of physiologically substances [2-5]. Previously these authors have destudied the mechanism of seed coating effect on the biochemical processes underlying in their germination. On the corn shoots stage in the variants, where the seeds have been treated with improved multicomponent physiologically active polyfunctional (nano) chips with herbicide activity, rice plants height was higher than in the control variant. A similar tendency was observed in root length indices.

On the stooling stageplant stand density varied widely. Furthermore, on the same stage polyfunctional, multicomponent, complex (nano) chips with herbicide activity developed for pre-sowing rice seed treatment also led to the increase of plant stand density when compared to the control variant. Similar tendencies were observed in rice plant height and root length indices.

On the booting stage in all the variants, where the seeds have been treated with developed multicomponent, polyfunctional (nano) chips that posses herbicide activity, plant stand density index was also higher than the control indexes and had the maximum values if compared to other experiment variants.

On the booting stage the height index of the rice plants treated with efficient, multicomponent, polyfunctional (nano) chips based on physiologically active substances was higher in comparison with the control variant as well as plants' root length index.

On the stooling stage plant stand density index varied widely. On this stage improved polyfunctional, multicomponent, complex (nano) chips for pre-sowing seed treatment also enabled plant stand density increase in comparison with the control variant. Plant root length index showed similar results.

On the booting stage in all the variants, where the seeds have been treated with developed multicomponent, physiologically active, polyfunctional (nano) chips with herbicide activity, rice stand density index was also higher than the control variants and was higher than the etalons (in this variant at rice sowing a herbicide has been used on the 3-4 leafs stage according to the recommendations of American company manufacturer "Dao Agrosciences").

On the booting stage the height of rice plants treated with effective developed multicomponent, polyfunctional (nano) chips based on physiologically active substances was higher if compared to both the control variant and the etalon variant. Furthermore, rice plants root length was also higher than the control and etalon values on the stage of development.

Studying the influence of developed polyfunctional (nano) systems with herbicide activity on weed control has shown that on the $15^{\text {th }}$ day the number of weeds (barnyard-grass and sedge species) in the experiment variants was the least and depended on the structure of (nano) systems with different components in various combinations (NaKMC, modified vermiculite MVM, elicitor AgroHit, herbicide $\mathrm{H}$ ). 
On the $30^{\text {th }}$ day under the influence of the developed (nano) systems, weed control index was slightly lower in comparison with the indices obtained on the $15^{\text {th }}$ day, but was significantly higher than the control values.

On the $60^{\text {th }}$ day developed polyfunctional (nano) chips with herbicide activity were also efficient at weed control in rice. The peak values of weed control indices were $87,0 \%$ for sedge species and $84.9 \%$ for barnyard-grass species.

Examining rice development biometrical data allowed us to conclude that plant stand density and plant height indices were higher than the control values by the end of the vegetation period due to the influence of our developed multicomponent, polyfunctional (nano) chips with herbicide activity. However, stooling coefficient varied widely depending on the experiment variant, the highest stooling coefficient has been recorded in the variants with the seed treatment that included NaKMC together with AgroHit, MVM and a herbicide (multicomponent, polyfunctional (nano) chips with biopesticide and herbicide additions) or NaKMC with AgroHit and a herbicide $\mathrm{H}$.

The highest major panicle length has been recorded in the variants where the seeds had been treated with multicomponent, polyfunctional (nano) chips with herbicide activity (NaKMC along with AgroHit and herbicide $\mathrm{H}$ ).

The highest major panicle seed weight has been recorded in the variant where the seeds had been treated with the (nano) chip that included NaKMC, AgroHit, MVM and herbicide $\mathrm{H})$. This variant showed the increase of side panicle seed weight when compared to the control, etalon and other variants of the experiment. Besides, hollow seed index was the least in the variant where the seeds had been treated with multicomponent, polyfunctional (nano) chips of the same structure with herbicide activity (NaKMC along with AgroHit, MVM and herbicide H). 1000 seed weight in all the variants was higher than the control values and reached its maximum in the variant where the seeds had been treated with NaKMC, AgroHit, MVM and herbicide $\mathrm{H}$ or with NaKMC, AgroHit and herbicide $\mathrm{H}$.

In comparison with the control and etalon variants the highest yield increase has been observed in the variant where the seeds had been treated during the pre-sowing period with the developed multicomponent, polyfunctional, complex (nano) chip with herbicide activity which included MVM, NaKMC, AgroHit and herbicide H, the increase made up 19,0 clha.

Thus, developed multicomponent, physiologically active, polyfunctional (nano) systems for pre-sowing rice seed treatment, possessing herbicide activity, considerably increase crop yield when compared to the control or etalon variants and also enable weed control in rice. Combined activities of developed (nano) chip components, in various combinations and at different ratios, contribute to the results obtained. It should be also noted that the researched agrotechnology may promote a stable development of rice and other crops sowing seeds production, it may also aid plant breeding and agricultural sector development in general. This becomes possible since this agrotechnology presupposes the usage of (nano) chips with physiologically active substances that have a wide action spectrum and labile, mobile composition and features which, according to our forecasts, can change. Application of biopesticides as a part of (nano) chips allows obtaining environmentally sound products and in the future - "nanoproducts".

According to the conventional scientific terminology, a product can be called a "nanoproduct", if nanoparticles, nanotechnological development or instrument have been used at its sowing, manufacturing, processing or packaging. Nanoproducts engineers aim at food production advancement, its taste and nutritional values improvement, at "functional" products manufacturing and at increasing production level and lowering prices on food. 
As it has been noted in the report prepared for British Royal Research Society in already couple of decades "nanoproducts" application will be universal.

"Nanoproduct" research scale is impressive as well as the rate of investments attracted. In the recent years the world's leading food manufacturers such as Kraft, Nestle, Heinz, Altria, Unilever, have invested vast sums of money in nanotechnologies development. According to the recent estimation the value of nanoproducts market goes up to 410 million dollars and by the year 2012 an increase to 5,8 billion dollars is expected. Various research centers of not only the developed industrialized countries, but also of the developing countries carry out research works in the sphere of " nanoproducts". In the agricultural sector (nano) technologies are also being introduced, these technologies are aimed at boosting environmentally safe goods production and, finally, at "nanoproducts" manufacturing.

\section{CONCLUSIONS}

1. In this research work we have developed and introduced the agro (nano) technology for pre-sowing treatment of rice seeds with environmentally sound physiologically active polyfunctional multicomponent complex (nano) chips with herbicide activity. These (nano) chips are based on natural minerals and polysaccharide derivatives from local raw material recourses: modified vermiculite MVM, sodium salt of carboxymethylcellulose $\mathrm{NaCMC}$, plant protection means (a herbicide and a biopesticide - AgroHit elicitor).

2. We have carried out the necessary in-vitro experiments on determining the efficiency of (nano) systems for the development of polyfunctional, multicomponent, complex, physiologically active (nano) chips for pre-sowing seed treatment.

3. It has been shown that weed control efficiency in rice was on the level of the etalon values or close to them in all the variants where environmentally safe polyfunctional, multicomponent, complex (nano) chips with herbicide activity had been applied. Such (nano) chips were based on modified vermiculite MVM, NaCMC, elicitor AgroHit and the herbicide and put on the seed surface during the pre-sowing treatment (the plant were treated on the 3-4 leaf stage according to the manufacturing company recommendations).

4. It has been also determined that the major yield increase to $19,0 \mathrm{c} \backslash$ ha relative to the control variant (where the seeds were not treated) and to $10,8 \mathrm{c}$ ha relative to the etalon variant (where the plants were treated with herbicide on the 3-4 leaf stage according to the manufacturing company recommendations) occurred at pre-sowing treatment with environmentally sound, polyfunctional, multicomponent (nano) chips with herbicide activity. The (nano) chips were based on modified vermiculite MVM with $\mathrm{NaCMC}$, elicitor AgroHit and herbicide $\mathrm{H}$.

5. The regulations have been introduced regarding per-sowing seed treatment with (nano) technologies including polyfunctional, multicomponent, physiologically active (nano) chips based on modified natural mineral MVM and modified natural polysaccharide derivatives (AgroHit, sodium salt of carboxymethylcellulose NaCMC and the herbicide). 


\section{References}

[1] Nizhegorodov U.A. Vermiculite and Vermiculite Technology. - Irkutsk:, Russia Business-Story, (2008). 500.

[2] N. Voropaeva, V. Karpachev, V. Gorshkov, V. Varlamov, O. Figovsky, J. Scientific Israel - Technological Advantages 15(2) (2013) 72-78.

[3] N. Voropaeva, V. Karpachev, V. Varlamov, O. Figovsky, J. Scientific Israel Technological Advantages 15(1) (2013) 79-82.

[4] Zaharenko V. A., J. Plant protection and quarantine N1 (2013) 20.

[5] Ruban I. N., Voropaeva N. L, Figovsky O. L. et.al. Patent USA 2459518. (2012). 